\title{
New Perception of the Optical Solutions to the Fokas-Lenells Equation According to Two Different Techniques
}

Emad Zahran ( $\boldsymbol{D}$ e_h_zahran@hotmail.com )

Benha University

Maha Shehata

Zagazig University

\section{Research}

Keywords: The Perturbed Fokas-Lenells Equation (FLE), The Modified Simple Equation Method (MSEM), The Modified Extended Tanh-Function Method (METFM), the varitional iteration method, Traveling Wave Solutions, The numerical solutions

Posted Date: July 23rd, 2020

DOI: https://doi.org/10.21203/rs.3.rs-41748/v1

License: (c) (i) This work is licensed under a Creative Commons Attribution 4.0 International License. Read Full License 


\title{
New perception of the Optical solutions to the Fokas-Lenells equation according to two different techniques
}

\author{
Emad H.M. Zahran ${ }^{1}$, Maha S.M .Shehata ${ }^{2}$ \\ ${ }^{1}$ Department of Mathematical and Physical Engineering, Benha University, Faculty of Engineing \\ Shubra, Egypt; \\ ${ }^{2}$ Department of Mathematics, Zagazig University, Faculty of Science, Zagazig, Egypt;
}

The corresponding author Email address:e_h_zahran@ hotmail.com

\begin{abstract}
In this article, the perturbed Fokas-Lenells equation (FLE)" which plays a vital role in modern asocial media and electronic communication" is employed. Two important different methods are invited to demonstrating new accurate solutions of this equation. The first method is the modified simple equation method (MSEM) that reduces large volume of calculations and realizes closed form solution. While the second is the modified extended tanh-function method (METFM) "which controlled by the auxiliary Ricatti equation" and used effectively to obtain accurate solutions Furthermore, few of the realized results are compatible with that obtained by previous authors elsewhere the others remains new. In addition to the varitional iteration method (VIM )is applied perfectly to achieved the numerical solution corresponding to the exact solution realized by each one of these methods individually.
\end{abstract}

Keywords : The Perturbed Fokas-Lenells Equation (FLE); The Modified Simple Equation Method (MSEM); The Modified Extended Tanh-Function Method (METFM); the varitional iteration method; Traveling Wave Solutions; The numerical solutions.

\section{Introduction}

The breakthrough of the soliton technology is clearly manifested in recent asocial media and modern electronic communications namely the internet blogs, Facebook communication, twitter comments". The suggested equation [1-5] represent this phenomenon can be written as:

$$
i p_{t}+a_{1} p_{x x}+a_{2} p_{x t}+\left|p^{2}\right|\left(\beta p+i \eta p_{x}\right)=i\left[\gamma p_{x}+\rho\left(|p|^{2 n}\right)_{x} p\right]
$$

The first term of Eq. (1.1) denotes for temporal development of the pulses, whereas the two independent variables $\mathbf{x}$ and $\mathbf{t}$ in the complex-valued function $\mathrm{p}(\mathrm{x}, \mathrm{t})$ represent the spatial and temporal components respectively. The variables in the left hand side are distinguished as $\beta$ is self -phase modulation, $\mu$ revise to the nonlinear dispersal, while $a_{1}, a_{2}$ are the group velocity dispersal and the spatial-temporal dispersals respectively. On the right hand side $\gamma$ is the influence of inter-modal dispersal that is in addition to chromatic dispersal and $\rho$ is the self-deepening effect while the coefficient $m$ gives the effect of nonlinear dispersal with full nonlinearity. Finely the parameter $\mathrm{n}$ is the full nonlinearity parameter.

The accurate solution to the NLPDEs which plays a crucial role in big varieties of biological, chemical and physical phenomena is one of the most exciting advances for looking it. The NLPDEs can introduce much physical information and more insight into the physical aspects of these phenomenon's and its applications. Recently, with the aid the swift advancement of 


\section{The corresponding author Email address:e_h_zahran@hotmail.com}

computer algebraic system the analytical solutions for the nonlinear partial differential equations (NPDEs) have been realized [6-14]. There are three principal axes to get the exact solutions to NLPDEs namely the reduction methods, Lie symmetry group and the ansatze approaches methods. The famous ansatze methods are demonstrated through [6-16]. The most famous and powerful one of these methods is the MSEM which significant by its concentrated and accuracy to describe briefly the closed form solutions for the nonlinear problems. There are some tries to study the soliton dynamics by solving FLE listed at [15-35]. There are a variety of mathematical procedures that make the study of soliton dynamics possible [31-35] proposed a new methods to address accurate solutions and present conservation laws to this model. Also, A.H. Kara,and others [35] have obtained the Optical soliton solution to this equation using the jacobi elliptic function and $\left(\mathrm{G}^{\prime} / \mathrm{G}\right)$-expansion method. Also the varitional iteration method [36] has been used effectively to obtain the numerical solution corresponding to the exact solution to satisfied by each one of these methods. Our target going to apply the MSEM and the (METFM) as a two new techniques to get exact and hence the solitary form solutions for the equation (1.1) mentioned above as well as the numerical solution using the VIM.

2. Closed form solution using the MSEM [23-24]:

This section is pending on the MSEM arising in [23-24] to get the closed form solution which contain some variables. If one give these variables definite values he will realize the solitary solution.

\section{Description of the method}

To propose the general forlasim of the nonlinear evolution equation, let us introduce $\mathrm{R}$ as a function of $h(x, t)$ and its partial derivatives as,

$$
R\left(F, F_{t}, F_{x}, F_{t t}, F_{x x}, \ldots \ldots\right)=0,
$$

that involve the highest order derivatives and nonlinear terms.

With the aid of the transformation $F(x, t)=F(\zeta), \quad \zeta=x-w t$ and $k$ is cons. equation (2.1) can be reduced to the following ODE:

$$
S\left(F, F^{\prime}, F^{\prime \prime}, F^{\prime \prime \prime}, \ldots \ldots\right)=0,
$$

where $S$ is a function in $F(\zeta)$ and its total derivatives, while $F^{\prime}=\frac{d F}{d \zeta}$.

\section{The MSEM admits these steps,}

Step1.

Suppose the solution of Eq. (2.2) according to the modified simple equation method as

$$
F(\zeta)=\sum_{k=0}^{n} A_{k}\left[\frac{\psi^{\prime}(\zeta)}{\psi(\zeta)}\right]^{k}
$$




\section{The corresponding author Email address:e_h_zahran@ hotmail.com}

Where $A_{k}$ are arbitrary constants to be determined, such that $A_{n} \neq 0$, while the function $\psi(\zeta)$ is unknown function to be determined later, such that $\psi^{\prime}(\zeta) \neq 0$.

Step2.

Determine the positive integer $n$ in Eq. (2.3) by balancing the highest order derivative term and the nonlinear term.

Step3.

From Eq.(2.3) by substituting about the derivative $\left(F^{\prime}, F^{\prime \prime}, \ldots \ldots.\right)$ of the function $F(\zeta)$, we get a polynomial of $\psi^{-j}(j=0,1,2, \ldots$.$) . In this polynomial, adding all terms of the same$ power of $\psi^{-j}$ and hence equating the coefficients of different powers of $\psi^{-j}$ to zero, we get a system of algebraic equations which solved to find $A_{k}$ and $\psi(\zeta)$.

\section{Application}

First of all, we introduce the following wave transformation,

$$
\begin{aligned}
& q(x, t)=F(\zeta) \exp i H(x, t), \zeta=x-w t \\
& H(x, t)=B(x-w t)
\end{aligned}
$$

with these restrictions $\sigma=3 \lambda-2 \mu, \alpha=-w+2 a_{1} B-2 w a_{2} B$, will represents the shape features of the wave pulse, and $H(x, t)$ is the phase element of the soliton.

Moreover for $\mathrm{m}=1$, Eq. (3.1) will reduce the perturbed Fokas-Lenells equation (1.1) to the following ordinary differential equation [40],

$$
\left(a_{1}-a_{2} w\right) F^{\prime \prime}+\left(a_{1}-a_{2} w\right) B^{2} F+(\beta-2 \lambda B+2 \mu B) F^{3}=0
$$

According to the modified simple equation method by balancing the nonlinear term and the higher order derivative term, we get $n=1$, hence the solution according to the constructed method is:

$$
F(\zeta)=A_{0}+A_{1}\left(\frac{\psi^{\prime}(\zeta)}{\psi(\zeta)}\right)
$$

Now, it is easy to obtain $F^{\prime \prime}$ as,

$$
F^{\prime}=A_{1}\left(\frac{\psi^{\prime \prime}}{\psi}-\left(\frac{\psi^{\prime}}{\psi}\right)^{2}\right), F^{\prime \prime}=A_{1}\left(\frac{\psi^{\prime \prime \prime}}{\psi}-\frac{3 \psi^{\prime} \psi^{\prime \prime}}{\psi^{2}}+2\left(\frac{\psi^{\prime}}{\psi}\right)^{3}\right),
$$

and hence, substitute about $F, F^{3}$ and $F^{\prime \prime}$ at Eq. (3.2), then equating the coefficients of different power of $\psi^{-j}$ to zero we get this system of algebraic equations, 


$$
\begin{aligned}
& 2\left(a_{1}-a_{2} w\right)+(\beta-2 \lambda B+2 \mu B) A_{1}^{3}=0, \\
& \left(a_{1}-a_{2} w\right) \psi^{\prime \prime}+A_{0} A_{1}^{2}(\beta-2 \lambda B+2 \mu B) \psi^{\prime}=0, \\
& \left(a_{1}-a_{2} w\right) \psi^{\prime \prime \prime}+\left[\left(a_{1}-a_{2} w\right) B^{2}\right. \\
& \left.+3(\beta-2 \lambda B+2 \mu B) A_{0}^{2}\right] A_{1} \psi^{\prime}=0, \\
& \left(a_{1}-a_{2} w\right) B^{2}+(\beta-2 \lambda B+2 \mu B) A_{0}^{2}=0,
\end{aligned}
$$

Solving this system of algebraic equations as:

(i) Firstly from the first and the fourth part of Eq. (3.4) by dividing we obtain,

$$
\frac{B^{2}}{2}=\frac{A_{0}^{2}}{A_{1}^{2}} \Rightarrow A_{1}=\sqrt[3]{\frac{3 A_{0}^{2}}{B^{2}}},
$$

(ii) Secondly, from the second and third part of Eq. (3.4) by dividing, we get,

$$
\frac{\psi^{\prime \prime}(\zeta)}{\psi^{\prime}(\zeta)}=\frac{A_{0} A_{1}^{2}(\beta-2 \lambda B+2 \mu B)}{\left(a_{2} w-a_{1}\right)} \zeta,
$$

Integrating once, we obtain:

$$
\psi^{\prime}(\zeta)=c_{1} \exp \left[\frac{A_{0} A_{1}^{2}(\beta-2 \lambda B+2 \mu B)}{\left(a_{2} w-a_{1}\right)}\right] \zeta,
$$

Integrating another once, we obtain;

$$
\psi(\zeta)=c_{2}+\frac{c_{1}\left(a_{2} w-a_{1}\right)}{A_{0} A_{1}^{2}(\beta-2 \lambda B+2 \mu B)} \exp \left[\frac{A_{0} A_{1}^{2}(\beta-2 \lambda B+2 \mu B)}{\left(a_{2} w-a_{1}\right)}\right] \zeta
$$

Thus, substitute at Eq. (3.3) we get,

$$
F(x, t)=A_{0}+A_{1}\left(\frac{c_{1} \exp \left[\frac{A_{0} A_{1}^{2}(\beta-2 \lambda B+2 \mu B)}{\left(a_{2} w-a_{1}\right)}\right](x-w t)}{c_{2}+\frac{c_{1}\left(a_{2} w-a_{1}\right)}{A_{0} A_{1}^{2}(\beta-2 \lambda B+2 \mu B)} \exp \left[\frac{A_{0} A_{1}^{2}(\beta-2 \lambda B+2 \mu B)}{\left(a_{2} w-a_{1}\right)}\right](x-w t)}\right),
$$

and hence, 


$$
\begin{gathered}
q(x, t)=\left[A_{0}+A_{1}\left(\frac{c_{1} \exp \left[\frac{A_{0} A_{1}^{2}(\beta-2 \lambda B+2 \mu B)}{\left(a_{2} w-a_{1}\right)}\right](x-w t)}{c_{2}+\frac{c_{1}\left(a_{2} w-a_{1}\right)}{A_{0} A_{1}^{2}(\beta-2 \lambda B+2 \mu B)} \exp \left[\frac{A_{0} A_{1}^{2}(\beta-2 \lambda B+2 \mu B)}{\left(a_{2} w-a_{1}\right)}\right](x-w t)}\right]\right] \exp i B(x-w t), \\
\operatorname{Re}(q(x, t))=A_{0} \cos (B(x-w t))+A_{1}\left(\frac{c_{1} \cos (B(x-w t)) \exp \left(\frac{A_{0} A_{1}^{2}(\beta-2 \lambda B+2 \mu B)}{\left(a_{2} w-a_{1}\right)}\right)(x-w t)}{c_{2}+\frac{c_{1}\left(a_{2} w-a_{1}\right)}{A_{0} A_{1}^{2}(\beta-2 \lambda B+2 \mu B)} \exp \left(\frac{A_{0} A_{1}^{2}(\beta-2 \lambda B+2 \mu B)}{\left(a_{2} w-a_{1}\right)}\right)(x-w t)}\right), \\
\operatorname{Im}(q(x, t))=A_{0} \sin (B(x-w t))+A_{1}\left(\frac{c_{1} \sin (B(x-w t)) \exp \left(\frac{A_{0} A_{1}^{2}(\beta-2 \lambda B+2 \mu B)}{\left(a_{2} w-a_{1}\right)}\right)(x-w t)}{c_{2}+\frac{c_{1}\left(a_{2} w-a_{1}\right)}{A_{0} A_{1}^{2}(\beta-2 \lambda B+2 \mu B)} \exp \left(\frac{A_{0} A_{1}^{2}(\beta-2 \lambda B+2 \mu B)}{\left(a_{2} w-a_{1}\right)}\right)(x-w t)}\right),
\end{gathered}
$$

\section{$\mathrm{t}=\mathbf{0 . 2}$}


Figure 1. the plot of Eq.(3.10) in 2D and 3D with values: $a_{1}=0.1, a_{2}=0.5, \mu=1, \lambda=1$, $\beta=1, w=2, B=0.3, c_{1}=0.8, c_{2}=1, A_{0}=0.9, A_{1}=3,-1 \mathrm{p} \times \mathrm{p} 1,0 \mathrm{p} t \mathrm{p} 20$ 
$\mathrm{t}=\mathbf{0 . 2}$


Figure 2. the plot of Eq.(3.11) in 2D and 3D with values: $a_{1}=0.1, a_{2}=0.5, \mu=1, \lambda=1$, $\beta=1, w=2, B=0.3, c_{1}=0.8, c_{2}=1, A_{0}=0.9, A_{1}=3,-1 \mathrm{p} \times \mathrm{p} 1,0 \mathrm{p} t \mathrm{p} 20$

\section{Accurate optical solutions using the modified extended tanh-function method [27-28]}

This section is composed on the modified extended tanh-function method [27-28] which is another technique (which depend on the balance rule) arising to get the exact solution in terms of some parameters. If these parameters take definite values the solitary wave solution is realized.

\section{Description of the method}

According to the general forlasim of the nonlinear evolution equation mentioned above in (2.1), (2.2) the constructed solution to METFM is:

$$
H(\zeta)=A_{0}+\sum_{i=1}^{n}\left(A_{i} \phi_{i}+\frac{B_{i}}{\phi_{i}}\right)
$$

The balance rule mentioned in [18], is used to evaluate the integer m arise in Eq. (4.1) such that either $A_{m} \neq 0$ or $B_{m} \neq 0$ while $A_{i}, B_{i}$ well be calculated. Also $\phi$ must satisfy the Riccati equation $\phi^{\prime}=b+\phi^{2}$, and according to the value of $\mathrm{b}$ three well known of solutions are admitted namely;

$$
\begin{aligned}
& \varphi=-\sqrt{-B} \tanh (\sqrt{-B} \zeta), \text { or } \quad \varphi=-\sqrt{-B} \operatorname{coth}(\sqrt{-B} \zeta), \quad B<0 \\
& \varphi=\sqrt{B} \tan (\sqrt{B} \zeta), \text { or } \varphi=-\sqrt{B} \cot (\sqrt{B} \zeta), \quad B>0 \\
& \varphi=-\frac{1}{\zeta}, \quad B=0
\end{aligned}
$$

Let the coefficients of different powers of $\phi^{i}$ in these solutions equal to zero, we can generate 
an algebraic system of equations that solving by any computer program to get the values of the required constants.

\section{Application:}

We will apply the modified extended tanh-function method for the equation (3.2) mentioned above as follow,

$$
F(\zeta)=A_{0}+A_{1} \phi_{1}+\frac{B_{1}}{\phi_{1}}
$$

Now, we substitute about $F^{\prime \prime}, F$ and $F^{3}$ at equation (3.2) and then equating the coefficients of different power of $F$ to zero we get this system of algebraic equations,

$$
\begin{gathered}
\varphi^{3} \Rightarrow \quad(\beta-2 \lambda B+2 \mu B) A_{1}^{3}=0 \\
\varphi^{2} \Rightarrow 3(\beta-2 \lambda B+2 \mu B) A_{0} A_{1}^{2}=0 \\
\varphi \Rightarrow\left(a_{1}-a_{2} w\right)\left(B^{2}\right)+(\beta-2 \lambda B+2 \mu B)\left(3 A_{0}^{2}+3 A_{1} B_{1}\right)=0, \\
\varphi^{-3} \Rightarrow-2\left(a_{1}-a_{2}\right) \varphi^{\prime 2}+(\beta-2 \lambda B+2 \mu B) B_{1}^{2}=0 \\
\varphi^{-2} \Rightarrow\left(a_{2}-a_{1}\right) \varphi^{\prime \prime}+3(\beta-2 \lambda B+2 \mu B) A_{0} B_{1}=0 \\
\varphi^{-1} \Rightarrow\left(a_{1}-a_{2} w\right) B^{2}+\left(3 A_{1} B_{1}+3 A_{0}^{2}\right)(\beta-2 \lambda B+2 \mu B)=0 \\
\text { Cons. } \Rightarrow\left(a_{1}-a_{2}\right) A_{1} \varphi^{\prime \prime}+\left(a_{1}-a_{2} w\right) A_{0} B^{2}+\left(6 A_{0} A_{1} B_{1}+A_{0}^{3}\right)(\beta-2 \lambda B+2 \mu B)=0
\end{gathered}
$$

We can easily solve this system of algebraic equations analytically as follow, equation (5.2) lead to $A_{1}=0$ consequently equations (5.9) and (5.12) implies,

$$
A_{0}= \pm B \sqrt{\frac{a_{2}-a_{1}}{3(\beta-2 \lambda B+2 \mu B)}},
$$

Dividing equation (5.6) by equation (5.5) and integrating once we obtain,

$$
B_{1}= \pm \sqrt{\frac{\left(a_{2}-a_{1}\right) e^{-12 A_{0} \sqrt{\beta-2 \lambda B+2 \mu B}}}{\beta-2 \lambda B+2 \mu B}}
$$

According to these obtained results we take only case (3), hence the solutions is, 


$$
\begin{gathered}
F(\zeta)=A_{0}+A_{1} \phi_{1}+\frac{B_{1}}{\phi_{1}} \\
F(\zeta)= \pm 0.3 \sqrt{\frac{0.4}{3(1-0.6 \lambda+0.6 \mu)}}+\frac{\sqrt{\frac{0.4 e^{ \pm 3.6 \sqrt{\frac{0.4}{3}}}}{1-0.6 \lambda+0.6 \mu}}}{\phi_{1}}
\end{gathered}
$$

Consequently

$$
\begin{aligned}
& q(x, t)= \pm \sqrt{\frac{0.4}{3(1-0.6 \lambda+0.6 \mu)}}+\frac{\sqrt{\frac{0.4 e^{ \pm 3.6 \sqrt{\frac{0.4}{3}}}}{1-0.6 \lambda+0.6 \mu}}}{\phi_{1}} \exp i B(x-w t), \\
& \operatorname{Re} q(x, t)= \pm \sqrt{\frac{0.4}{3(1-0.6 \lambda+0.6 \mu)}}+\frac{\sqrt{\frac{0.4 e^{ \pm 12 \sqrt{\frac{0.4}{3}}}}{1-0.6 \lambda+0.6 \mu}}}{\phi_{1}} \cos (B(x-w t)) \\
& \operatorname{Im} q(x, t)= \pm \sqrt{\frac{0.4}{3(1-0.6 \lambda+0.6 \mu)}}+\frac{\sqrt{\frac{0.4 e^{ \pm 12 \sqrt{\frac{0.4}{3}}}}{1-0.6 \lambda+0.6 \mu}}}{\phi_{1}} \sin (B(x-w t))
\end{aligned}
$$

The following two figures represent the real equation (5.14), $B=0.3$






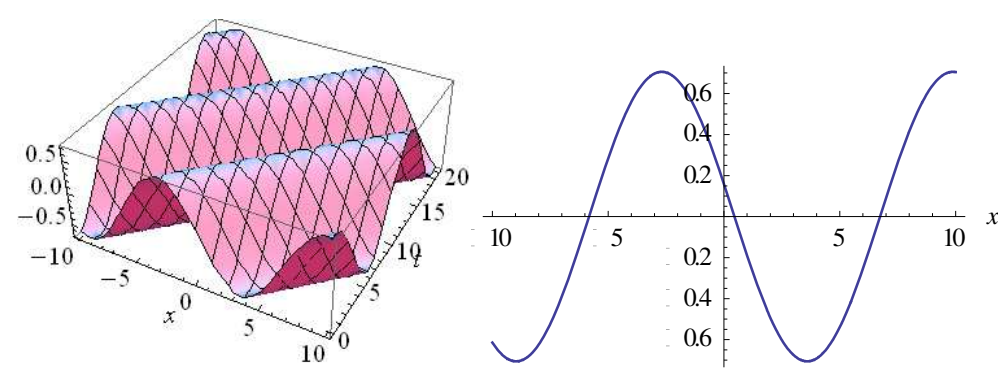

Figure 3. the plot of real part Eq.(5.14) in 2D and 3D with values:

$$
a_{1}=0.1, a_{2}=0.5, \mu=1, \lambda=1, \beta=1, w=1, B=0.3,-10<\mathrm{x}<10,0<\mathrm{t}<20
$$

The following two figures represent the imaginary part equation (5.15), $B=0.3$

$$
\mathrm{t}=\mathbf{0 . 2}
$$
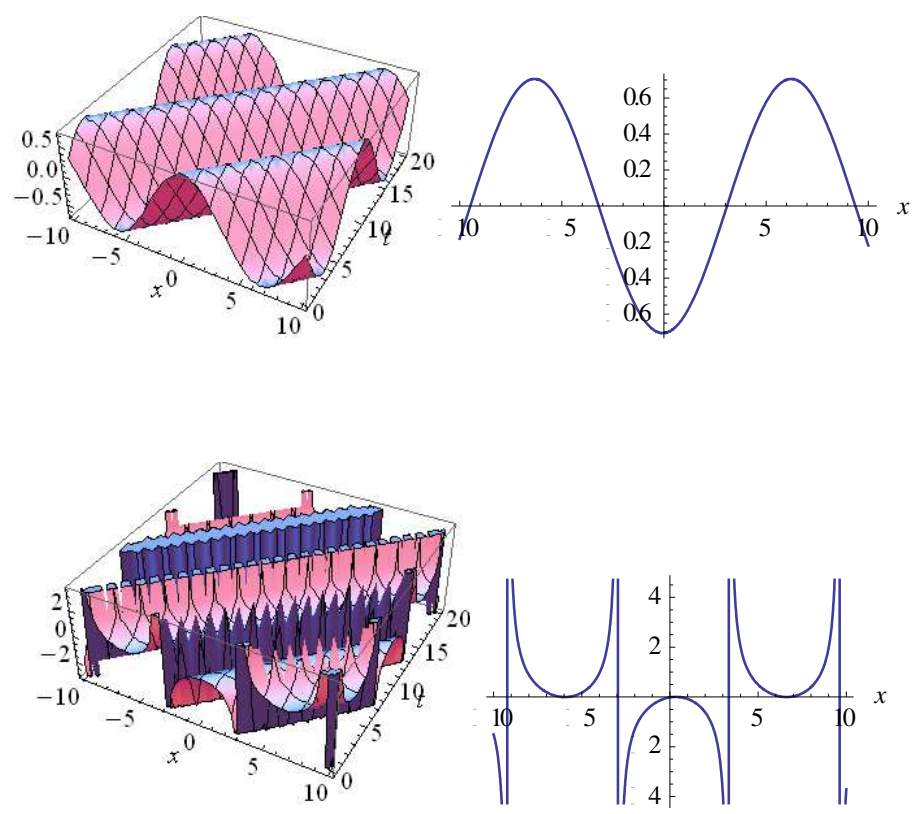

Figure 4. the plot of imaginary part Eq.(5.14) in 2D and 3D with values:

$$
a_{1}=0.1, a_{2}=0.5, \mu=1, \lambda=1, \beta=1, w=1, B=0.3,-10<\mathrm{x}<10,0<\mathrm{t}<20
$$

The following two figures represent the real equation (5.15), $B=-0.3$ 


\section{The corresponding author Email address:e_h_zahran@ hotmail.com}
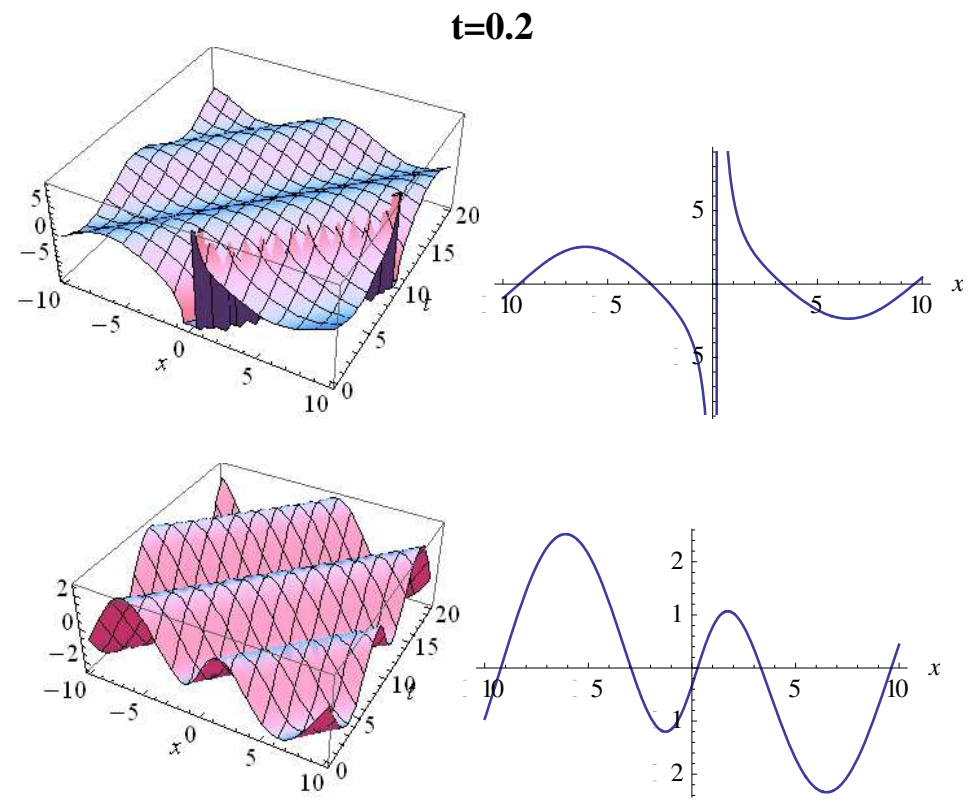

Figure 5. the plot of real part Eq.(5.15) in 2D and 3D with values:

$a_{1}=0.1, a_{2}=0.5, \mu=1, \lambda=1, \beta=1, w=1, B=-0.3,-10<\mathrm{x}<10,0<\mathrm{t}<20$

The following two figures represent the imaginary part equation (5.15), $B=-0.3$

$\mathrm{t}=\mathbf{0 . 2}$
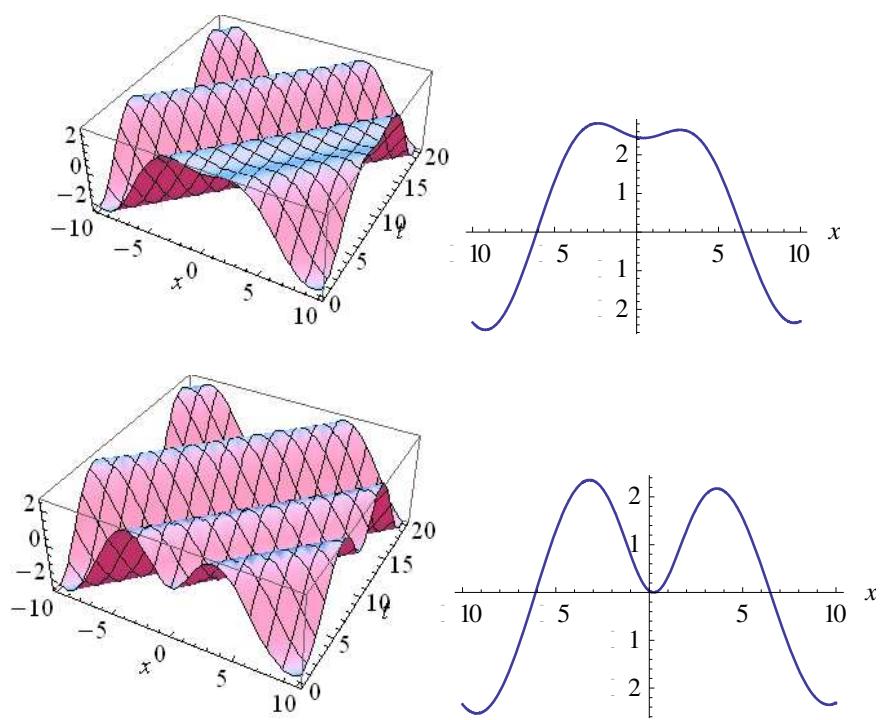

Figure 6. the plot of real part Eq.(5.15) in 2D and 3D with values:

$a_{1}=0.1, a_{2}=0.5, \mu=1, \lambda=1, \beta=1, w=1, B=-0.3,-10<\mathrm{x}<10,0<\mathrm{t}<20$ 


\section{The corresponding author Email address:e_h_zahran@ hotmail.com}

\section{The Variational iteration method [36]}

Consider the differential equation with inhomogeneous term $f(\xi)$ and $\mathrm{L}, \mathrm{N}$ the linear and the nonlinear operators respectively as;

$$
L h+N h=f(\xi),
$$

The VIM proposes a correction functional for equation (6.1) to be;

$$
h_{m+1}(\xi)=h_{m}(\xi)+\int_{0}^{\xi} \lambda(t)\left(L h_{m}(t)+N h_{m}^{o}(t)-g(t)\right) d t,
$$

where $\lambda$ is a general Lagrange's multiplier, which can be identified optimally via the variational theory, and $h_{m}^{o}$ as a restricted variation which means $\delta h_{m}^{o}$. The Lagrange multiplier $\lambda$ is crucial and critical in the method, and it can be a constant or a function. Having $\lambda$ determined, an iteration formula should be used for the determination of the successive approximations $h_{m+1}(\xi) ; n \geq 0$ of the solution $h(\xi)$. The zeros approximation $h_{0}$ can be any selective function. However, using the initial values $h(0) ; h^{\prime}(0)$ are preferably used for the selective zeros approximation $u_{0}$ as will be seen later. Consequently, the solution is given by $h(\xi)=\lim _{\xi \rightarrow \infty} h_{m}(\xi)$ It is interesting to point out that we formally derived the distinct forms of the Lagrange multipliers $\lambda$ in (6.1), hence we skip details. We only set a summary of the obtained results. It is important to give briefly the significant forms of Eq. (6.2) according to the Lagrange multipliers in these results.

For the 1-st order ODE in the form,

$$
h^{\prime}+q(\xi) h=p(\xi), h(0)=\rho,
$$

We find that $\lambda=-1$, and the correction function give the iteration formula;

$$
h_{m+1}(\xi)=h_{m}(\xi)-\int_{0}^{\xi}\left(h_{m}^{\prime}(t)+q(t) h_{m}(t)-p(t)\right) d t .
$$

The 2-nd order ODE in the form,

$$
h^{\prime \prime}(\xi)+c h^{\prime}(\xi)+d h(\xi)=g(\xi), \quad h(0)=\rho, h^{\prime}(0)=\eta,
$$

we find that $\lambda=t-x$, and the correction function give the iteration formula;

$$
h_{m+1}(\xi)=h_{m}(\xi)+\int_{0}^{\xi}(t-x)\left(h_{m}^{\prime \prime}(t)+c h_{m}^{\prime}(t)+d h_{m}-g(t)\right) d t .
$$


The 3-th order ODE in the form,

$$
h^{\prime \prime \prime}(\xi)+c h^{\prime \prime}(\xi)+d h^{\prime}(\xi)+e h(\xi)=g(\xi), h(0)=\rho, h^{\prime}(0)=\eta, h^{\prime \prime}(0)=\sigma,
$$

We find that $\lambda=-\frac{1}{2 !}(t-x)^{2}$, and the correction function give the iteration formula

$$
h_{m+1}(\xi)=h_{m}(\xi)-\frac{1}{2 !} \int_{0}^{\xi}(t-x)^{2}\left(h_{m}^{\prime \prime \prime}(t)+c h_{m}^{\prime \prime}(t)+d h_{m}^{\prime}(t)+e h_{m}-g(t)\right) d t,
$$

Consecountly,for the general form of ODE

$$
h^{(m)}+f\left(h^{\prime}, h^{\prime \prime}, h^{\prime \prime \prime} \ldots \ldots . ., h^{(m-1)}\right)=g(\xi), h(0)=\rho_{0}, h^{\prime}(0)=\rho_{1}, h^{\prime \prime}(0)=\rho_{2} \ldots . ., h^{m-1}(0)=\rho_{m-1},
$$

The lagrange multiplier $\lambda$ take the general form $\lambda=\frac{(-1)^{m}}{(m-1) !}(t-x)^{m-1}$, while the general form of iteration rule become,

$$
h_{m+1}(\xi)=h_{m}(\xi)+\frac{(-1)^{m}}{(m-1) !} \int_{0}^{\xi}(t-x)^{m-1}\left(h^{(m)}+f\left(h^{\prime}, h^{\prime \prime}, h^{\prime \prime \prime}, \ldots \ldots . ., h^{(m-1)}\right)-g(t)\right) d t,
$$

Furthermore the zeros approximation $h_{0}(\xi)$ can be selected perfectly to be,

$$
h_{0}(\xi)=h_{0}(0)+h^{\prime}(0) \xi+\frac{1}{2 !} h^{\prime \prime}(0) \xi^{2}+\frac{1}{3 !} h^{\prime \prime \prime}(0) \xi^{3} \ldots \ldots+\frac{1}{(m-1) !} h^{m-1}(0) \xi^{m-1}
$$

where $m$ is the order of the ODE

(6-a) Numerical solution corresponding to the exact solution realized using the MSEM

For the second order differential equation (3.2) mentioned above,

$$
\left(a_{1}-a_{2} w\right) F^{\prime \prime}+\left(a_{1}-a_{2} w\right) B^{2} F+(\beta-2 \lambda B+2 \mu B) F^{3}=0
$$

With the initial condition $\quad F(0)=A_{0}+A_{1}\left(\frac{c_{1}}{c_{2}+\frac{c_{1}\left(a_{2} w-a_{1}\right)}{A_{0} A_{1}^{2}(\beta-2 \lambda B+2 \mu B)}}\right)$,

$$
F(0)=0.9+\frac{2.4}{0.56}=5.2
$$




$$
\begin{gathered}
F^{\prime}(0)=A_{1} c_{1}\left(\frac{\left(c_{2}+\frac{c_{1}\left(a_{2} w-a_{1}\right)}{A_{0} A_{1}^{2}(\beta-2 \lambda B+2 \mu B)}\right)\left(\left(c_{1}-\frac{c_{1}^{2}\left(a_{2} w-a_{1}\right)}{A_{0} A_{1}^{2}(\beta-2 \lambda B+2 \mu B)}\right) \frac{A_{0} A_{1}^{2}(\beta-2 \lambda B+2 \mu B)}{c_{1}\left(a_{2} w-a_{1}\right)}\right)}{c_{2}^{2}+\frac{c_{1}^{2}\left(a_{2} w-a_{1}\right)^{2}}{A_{0}^{2} A_{1}^{4}(\beta-2 \lambda B+2 \mu B)^{2}}+\frac{2 c_{1} c_{2}\left(a_{2} w-a_{1}\right)}{A_{0} A_{1}^{2}(\beta-2 \lambda B+2 \mu B)}}\right) \\
F^{\prime}(0)=0.3\left(\frac{4.3 \times 0.4 \times 1.8}{1+\frac{1.2}{26.2}+0.8}\right)=0.3 \frac{3.1}{1+0.05+0.8}=0.5
\end{gathered}
$$

According to the variational iteration method the first iterations is,

$$
\begin{gathered}
F_{0}(\zeta)=F(0)+\zeta F^{\prime}(0), F_{0}(\zeta)=5.2+0.5 \zeta \\
F_{1}(\zeta)=F_{0}(\zeta)-\int_{0}^{\zeta}\left(\left(a_{1}-a_{2} w\right) F_{0}^{\prime \prime}(t)+\left(a_{1}-a_{2} w\right) B^{2} F_{0}(t)+(\beta-2 \lambda B+2 \mu B) F_{0}^{3}(t)\right) d t \\
F_{1}(\zeta)=5.2+0.5 \zeta-\int_{0}^{\zeta}\left(-0.9(0.3)^{2}(5.2+0.5 t)-0.2(5.2+0.5 t)^{3}\right) d t \\
F_{1}(\zeta)=5.2+0.5 \zeta-\int_{0}^{\zeta}\left(-0.42-0.04 t-28.1-8.1 t-0.78 t^{2}-0.025 t^{3}\right) d t \\
F_{1}(\zeta)=5.2+29.02 \zeta+4.07 \zeta^{2}+0.26 \zeta^{3}+0.006 \zeta^{4} \\
q_{1}(\zeta)=\left(5.2+29.02 \zeta+4.07 \zeta^{2}+0.26 \zeta^{3}+0.006 \zeta^{4}\right) \operatorname{Expi}[0.3(x-2 t)]
\end{gathered}
$$$$
\operatorname{Re} q_{1}(\zeta)=\left(5.2+29.02 \zeta+4.07 \zeta^{2}+0.26 \zeta^{3}+0.006 \zeta^{4}\right) \operatorname{Cos}[0.3(x-2 t)]
$$$$
\operatorname{Im} q_{1}(\zeta)=\left(5.2+29.02 \zeta+4.07 \zeta^{2}+0.26 \zeta^{3}+0.006 \zeta^{4}\right) \operatorname{Sin}[0.3(x-2 t)]
$$ 

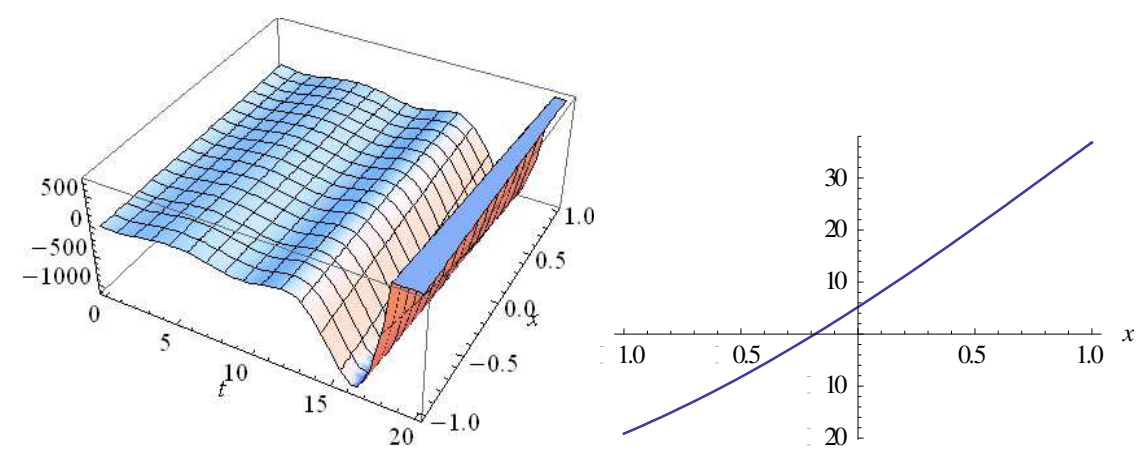

Figure 7. the plot of real part Eq.(7.4) in 2D and 3D with values: $a_{1}=0.1, a_{2}=0.5$, $\mu=1, \lambda=1, \beta=1, w=2, B=0.3, c_{1}=0.8, c_{2}=1, A_{0}=0.9, A_{1}=3,-1 \mathrm{p} x \mathrm{p} 1,0 \mathrm{p} t \mathrm{p} 20$
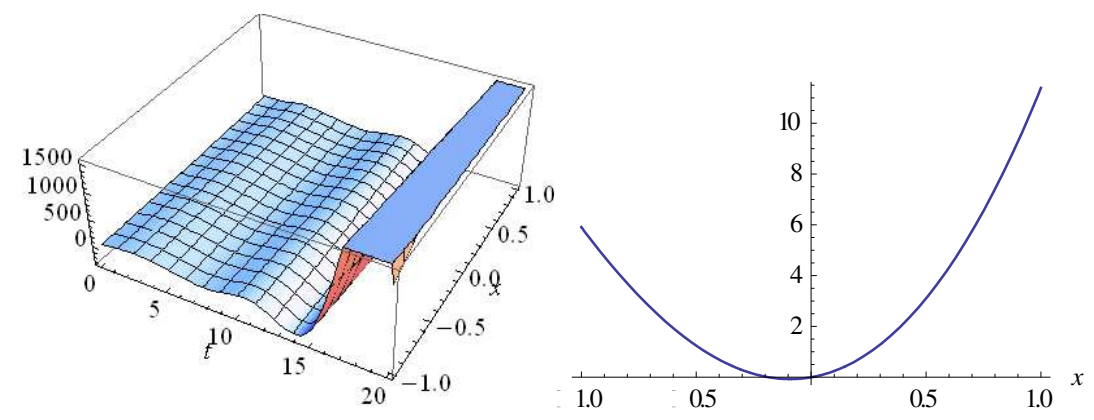

Figure 8. the plot of imaginary part Eq.(7.5) in 2D and 3D with values: $a_{1}=0.1, a_{2}=0.5$, $\mu=1, \lambda=1, \beta=1, w=2, B=0.3, c_{1}=0.8, c_{2}=1, A_{0}=0.9, A_{1}=3,-1 \mathrm{p} x \mathrm{p} 1,0 \mathrm{p} t \mathrm{p} 20$

(6-b) Numerical solution corresponding to the exact solution realized using the METFM

For the second order differential equation (3.2) mentioned above,

$$
\left(a_{1}-a_{2} w\right) F^{\prime \prime}+\left(a_{1}-a_{2} w\right) B^{2} F+(\beta-2 \lambda B+2 \mu B) F^{3}=0
$$

According to the obtained exact solutions generating by the METFM, the solution are either

$$
F(\zeta)=0.09-0.7 \tan (\zeta) \text { or } F(\zeta)=0.09-0.7 \tanh (\zeta)
$$

Consequently the first iterations according to the VIM for these two cases are the same, 


$$
\begin{aligned}
& F(0)=0.09, F^{\prime}(0)=-0.7, F_{0}(\zeta)=F(0)+F^{\prime}(0) \zeta=0.09-0.7 \zeta \\
& F_{1}(\zeta)=F_{0}(\zeta)-\int_{0}^{\zeta}\left(\left(a_{1}-a_{2} w\right) F_{0}^{\prime \prime}(t)+\left(a_{1}-a_{2} w\right) B^{2} F_{0}(t)+(\beta-2 \lambda B+2 \mu B) F_{0}^{3}(t)\right) d t \\
& F_{1}(\zeta)=0.09-0.7 \zeta-\int_{0}^{\zeta}\left(-0.4(0.3)^{2}(0.09-0.7 t)+1.6(0.09-0.7 t)^{3}\right) d t, \\
& F_{1}(\zeta)=0.09-0.7 \zeta-\int_{0}^{\zeta}\left(-0.003+0.03 t+0.001-0.03 t+0.21 t^{2}-0.55 t^{3}\right) d t \\
& F_{1}(\zeta)=0.09-0.696 \zeta-0.07 \zeta^{3}+0.14 \zeta^{4} \\
& q_{1}(\zeta)=\left(0.09-0.696 \zeta-0.07 \zeta^{3}+0.14 \zeta^{4}\right) \operatorname{Exp} i[0.3(x-t)] \\
& \operatorname{Re} q_{1}(\zeta)=\left(0.09-0.696 \zeta-0.07 \zeta^{3}+0.14 \zeta^{4}\right) \operatorname{Cos}[0.3(x-t)] \\
& \operatorname{Im} q_{1}(\zeta)=\left[0.09-0.696 \zeta-0.07 \zeta^{3}+0.14 \zeta^{4}\right) \operatorname{Sin}[0.3(x-t)]
\end{aligned}
$$
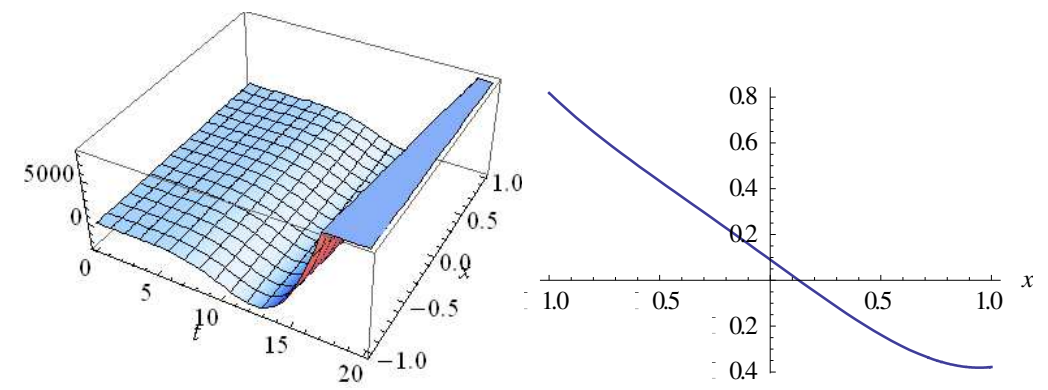

Figure 9. the plot of real part Eq.(7.7) in 2D and 3D with values: $a_{1}=0.1, a_{2}=0.5, \mu=1$,

$$
\lambda=1, \beta=1, w=1, B=0.3, A_{0}=0.09, B_{1}=0.35,-1 \mathrm{p} x \mathrm{p} 1,0 \mathrm{p} t \mathrm{p} 20
$$



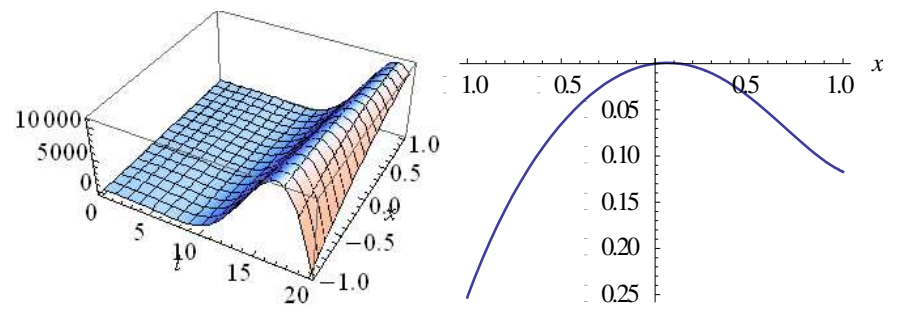

Figure 10. the plot of imaginary part Eq.(7.8) in 2D and 3D with values: $a_{1}=0.1, a_{2}=0.5$, $\mu=1, \lambda=1, \beta=1, w=1, B=0.3, a_{1}=0.1, a_{2}=0.5, A_{0}=0.09, B_{1}=0.35,-1 \mathrm{p} \times \mathrm{p} 1,0 \mathrm{p} t \mathrm{p} 20$

In addition to, the successive iteration for the all cases mentioned above can be calculated as,

$$
\begin{aligned}
& F_{2}(\zeta)=F_{1}(\zeta)-\int_{0}^{\zeta}\left(\left(a_{1}-a_{2} w\right) F_{1}^{\prime \prime}(t)+\left(a_{1}-a_{2} w\right) B^{2} F_{1}(t)+(\beta-2 \lambda B+2 \mu B) F_{1}^{3}(t)\right) d t, \\
& F_{3}(\zeta)=F_{2}(\zeta)-\int_{0}^{\zeta}\left(\left(a_{1}-a_{2} w\right) F_{2}^{\prime \prime}(t)+\left(a_{1}-a_{2} w\right) B^{2} F_{2}(t)+(\beta-2 \lambda B+2 \mu B) F_{2}^{3}(t)\right) d t, \\
& F_{m+1}(\zeta)=F_{m}(\zeta)-\int_{0}^{\zeta}\left(\left(a_{1}-a_{2} w\right) F_{m}^{\prime \prime}(t)+\left(a_{1}-a_{2} w\right) B^{2} F_{m}(t)+(\beta-2 \lambda B+2 \mu B) F_{m}^{3}(t)\right) d t
\end{aligned}
$$

Using the fact that the exact solution is obtained by using $F(\zeta)=\lim _{\zeta \rightarrow \infty} F_{m}(\zeta)$

\section{Captions, citations and physical meaning of the figures}

We can briefly give the captions and citations for the obtained figures instead of the corresponding physical meaning. Let us start from the results achieved by MSEM and the corresponding Figures $(1,2)$ which represents the periodic singular dark soliton solution to the equations (3.10),(3.11) respectively in two and three dimensions. Furthermore, the results realized by the METFM and the corresponding Figures (3-6) in two and three dimensions which are periodic bright and dark soliton solution. Most of achieved solutions using these two signfinacint methods weren't satisfied before. While Figures (7-10) represents the numerical solution obtained using VIT which derived its initial conditions from the realized exact solutions for each one of the proposed methods individually which are hyperbolic soliton solution. 


\section{The corresponding author Email address:e_h_zahran@hotmail.com}

\section{Results and Discussion}

The main target of this section is to give some graphical representations of the solutions achieved for the FLE. in 2D and 3D with values:

$a_{1}=0.1, a_{2}=0.5, \mu=1, \lambda=1, \beta=1, w=2, B=0.3, c_{1}=0.8, c_{2}=1, A_{0}=0.9, A_{1}=3$,

$-1 \mathrm{p} x \mathrm{p} 1,0 \mathrm{p} t \mathrm{p} 20$ for the MSEM while $t=0.2$ for 2D Figures (1-2).

While Figures (3-6) give the graphical representations of the solutions achieved using

METFM for the values: $a_{1}=0.1, a_{2}=0.5, \mu=1, \lambda=1, \beta=1, w=1, B=-0.3,-10<\mathrm{x}<10$, $0<\mathrm{t}<20$ as well as $t=0.2$ in the case 2D.

Also, Figures (7-9) give the graphical representations of the achieved solutions using VIM with the values,

$a_{1}=0.1, a_{2}=0.5, \mu=1, \lambda=1, \beta=1, w=1, B=0.3, A_{0}=0.09, B_{1}=0.35,-1 \mathrm{p} \times \mathrm{p} 1$,

$0 \mathrm{p} t \mathrm{p} 20$, for $t=0.2 \mathrm{in}$ case 2D. The obtained curves represent the amplitudes of the solutions and the solitary waves, particularly kink and anti-kink soliton solutions. These figures a good perception for the dynamics of the perturbed Fokas-Lenells equation represent several domains of social media specially, twitter, Internet blogs, Facebook communication as well as other the electronic communications,. Thus, the FLE possess a great importance in the nonlinear research. In fact that the FLE include rich physical features in solitary wave's theory and optical fibers phenomena. In addition, the outcomes of this work are new results which weren't realized in the literature.

\section{Conclusion}

In this work, the modified simple equation method has been applied effectively to realize the closed form solution for the first time to the perturbed Fokas-Lenells equation (FLE) Figure 1, Figure 2 which is positive forward future studies. Also, the modified extended tanh-function method has been applied successfully to obtain new accurate optical solutions Figures( 3-6) for this model. In addition to VIT which derived its initial conditions from the realized exact solutions for each one of the proposed methods individually has been success to obtained numerical solution corresponding to the obtained exact solutions figures (7-10). We also conclude that these constructed techniques admit new accurate effective solutions compared with that obtained by the previous work [33] and can be applied to other NLPDEs. Also the achieved numerical solution is more accurate than that obtained previously by other numerical methods. 


\section{List of Abbreviations}

\begin{tabular}{|l|l|l|}
\hline 1 & The perturbed Fokas-Lenells equation & FLE \\
\hline 2 & The modified simple equation method & MSEM \\
\hline 3 & The modified extended tanh-function method & METFM \\
\hline 4 & The varitional iteration method & VIM \\
\hline 5 & Equation & Eq. \\
\hline 6 & Two dimensions & 2D \\
\hline 7 & Three dimensions & $3 \mathrm{D}$ \\
\hline
\end{tabular}

\section{Declarations}

(11-a).Availability of supporting data: "Not applicable"

(11-b).Competing interests: This research didn't get any grant from any funding agency.

(11-c).Funding: There is no any funding interest.

(11-d).Authors contributions: All the research work in this paper is built by the authors.

\section{Reference}

[1] Biswas A, Yildirim Y, Yasar E, Zhou Q, Mahmood MF, Moshokoa SP, Belic M.; (2018), Optical solitons with differential group delay for coupled Fokas-Lenells equation using two integration schemes. Optik; 165; 74-86.

[2] Biswas A, Ekici M, Sonmezoglu A, Alqahtani RT.; (2018), Optical solitons with differential group delay for coupled Fokas-Lenells equation by extended trial function scheme. Optik ; 165; 102-10.

[3] Biswas A, Rezazadeh H, Mirzazadeh M, Eslami M, Ekici M, Zhou Q,Moshokoa SP, Belic M.; (2018), Optical soliton perturbation with Fokas-Lenells equation using three exotic and efficient integration schemes. Optik;165; 288-94.

[4] Jawad AJM, Biswas A, Zhou Q, Moshokoa SP, Belic M.; (2018), Optical soliton perturbation of Fokas-Lenells equation with two integration schemes. Optik;165; 111-16.

[5] Wazwaz AM. (2001), Construction of soliton solutions and periodic solutions of the Boussinesq equation by the modified decomposition method. Chaos Solitons Fractals;12; 1549-56.

[6] Gu, C.H. (1995),Soliton Theory and Its Application, Springer, Berlin.

[7] Kudryashov, N. A. (1991), On types of nonlinear non-integrable equations with exact solutions, Phys. Lett. A 155; 269-275.

[8] Zhang, H. (2007), Extended Jacobi elliptic function expansion method and its application, Commun. Nonlinear Sci. Numer. Simul. 12; 627-635.

[9] Kaur,L., Gupta, R.K.,(2014),Some invariant solutions of field equations with axial symmetry for empty space containing an electrostatic field, Appl. Math. Comp. 231; 560 


\section{The corresponding author Email address:e_h_zahran@hotmail.com}

565.

[10] Sun,B., Wazwaz, A.M.; (2018), General high-order breathers and rogue waves in thedimensional KP-Boussinesq equation, Commun. Non. Sci. Numer. Simul. 64; 1-13.

[11] Wazwaz, A.M. (2018),Painlevé analysis for a new integrable equation combining the modified Calogero-Bogoyavlenskii-Schiff (MCBS) equation with its negative-order form, Nonlinear Dyn. 91; 877-883.

[12] Kaur,L., Wazwaz, A.M.;(2018),Dynamical analysis of lump solutions for $(3+1)$ dimensional generalized KP-Boussinesq equation and its dimensionally reduced equations, Phys. Scr., https://doi.org/10.1088/1402-4896/aac8b8.

[13] Jawad, A. J. A. M.; (2012),New exact solutions of nonlinear partial differential equations using Tan-Cot function method. Studies in Mathematical sciences, 5(2);13-25.

[14] Biswas, A., Rezazadeh, H., Mirzazadeh, M., Eslami, M., Zhou, Q., Moshokoa, S. P., \& Belic, M.; (2018),Optical solitons having weak non-local nonlinearity by two integration schemes. Optik, 164; 380-384.


Some Nonlinear Evolution Equations in Mathematical Physics. American Journal of Computational Mathematics, 5(04), 468.

[16] Mirzazadeh, M., Eslami, M., \& Biswas, A.; (2014), Soliton solutions of the generalized Klein-Gordon equation by using $\left(\mathrm{G}^{\prime} / \mathrm{G}\right)$-expansion method. Computational and Applied Mathematics, 33(3); 831-839.

[17] Zahran, E. H., \& Khater, M. M.; (2014), Exact solution to some nonlinear evolution equations by the $\left(\mathrm{G}^{\prime} / \mathrm{G}\right)$-expansion method. Jokull journal, 64; 226-238.

[18] Rezazadeh, H., Mirhosseini-Alizamini, S. M., Eslami, M., Rezazadeh, M., Mirzazadeh, M., \& Abbagari, S.; (2018), New optical solitons of nonlinear conformable fractional Schrödinger-Hirota equation. Optik, 172; 545-553.

[19] Eslami, M., \& Rezazadeh, H.; (2016), The first integral method for Wu-Zhang system with conformable time-fractional derivative. Calcolo, 53(3); 475-485.

[20] Zahran, E. H., \& Khater, M. M.; (2014), Exact traveling wave solutions for the system of shallow water wave equations and modified Liouville equation using extended Jacobian elliptic function expansion method. American Journal of Computational Mathematics; 4(05), 455.

[21] Shehata, M. S.; (2015),Extended Jacobian Elliptic Function Expansion Method and its Applications for Solving some Nonlinear Evolution Equations in Mathematical Physics. International Journal of Computer Applications, 109(12); 1-4.

[22] Eslami, M., \& Mirzazadeh, M.; (2016), Optical solitons with Biswas-Milovic equation for power law and dual-power law nonlinearities. Nonlinear Dynamics, 83(1-2); 731-738.

[23] Zahran, E. H., \& Khater, M. M.; (2014), Exact traveling wave solutions for the system of shallow water wave equations and modified Liouville equation using the modified simple equation method.Jokull Journal,Vol.64 Issue 5.

[24] Khater, M. M., Zahran, E. H. \& Shehata, M. S. (2017). Solitary wave solution of the generalized Hirota-Satsuma coupled KdV system, Journal of Egyptian Mathematical Society, 25, 8-12.

[25] Yang, X. F., Deng, Z. C., \& Wei, Y.; (2015) A Riccati-Bernoulli sub-ODE method for nonlinear partial differential equations and its application. Advances in Difference Equations; (1); 117. 


\section{The corresponding author Email address:e_h_zahran@hotmail.com}

[26] Shehata, M. S., (2016), A new solitary wave solution of the perturbed nonlinear Schrodinger equation using a Riccati-Bernoulli Sub-ODE method. International Journal of Physical Sciences, 11(6); 80-84.

[27] Zahran, E. H., \& Khater, M. M.; (2016), Modified extended tanh-function method and its applications to the Bogoyavlenskii equation. Applied Mathematical Modelling, 40(3); $1769-1775$.

[28] Khater, M. M., Lu, D., \& Zahran, E. H.; (2017), Solitary wave solutions of the Benjamin-Bona-Mahoney-Burgers equation with dual power-law nonlinearity. Appl. Math. Inf. Sci, 11(5);1-5.

[29] Eslami, M.; (2016), Trial solution technique to chiral nonlinear Schrodinger's equation in (1+2)-dimensions. Nonlinear Dynamics, 85(2); 813-816.

[30] Rezazadeh, H., Korkmaz, A., Eslami, M., Vahidi, J., \& Asghari, R.; (2018), Traveling wave solution of conformable fractional generalized reaction Duffing model by generalized projective Riccati equation method. Optical and Quantum Electronics, 50(3); 150.

[31] Khodadad, F. S., Nazari, F., Eslami, M., \& Rezazadeh, H.; (2017), Soliton solutions of the conformable fractional Zakharov-Kuznetsov equation with dual-power law nonlinearity. Optical and Quantum Electronics, 49(11); 384 .

[32] Zhou, Q., Ekici, M., Sonmezoglu, A., Mirzazadeh, M., \& Eslami, M.; (2016), Optical solitons with Biswas-Milovic equation by extended trial equation method. Nonlinear Dynamics, 84(4); 1883-1900.

[33] Biswas A, Ekici M, Sonmezoglu A, Alqahtani RT.; (2018), Optical soliton perturbation with full nonlinearity for Fokas-Lenells equation. Optik; 165; 29-34.

[34] Wazwaz AM.; (2016), Gaussian solitary wave solutions for nonlinear evolution equations with logarithmic nonlinearities. Nonlinear Dyn;83; 591-596.

[35] Anupma Bansal , A.H. Kara, Anjan Biswas, Seithuti P. Moshokoa, Milivoj Belic, (2018), Optical soliton perturbation, group invariants and conservation laws of perturbed FokasLenells equation, Chaos, Solitons and Fractals 114; 275-280.

[36] Yusufoglu, E.; Bekir, A.; The variational iteration method for Solitary potterns solutions of gBBE equation, Physical Letter A; (2007);367, 6; 461-464.

\section{Figure Legends:}

Figure 1. the plot 3D and 2D surfaces of Eq. (3.10) by considering the values:

$a_{1}=0.1, a_{2}=0.5, \mu=1, \lambda=1, \beta=1, w=2, B=0.3, c_{1}=0.8, c_{2}=1, A_{0}=0.9, A_{1}=3$,

$-1 \mathrm{p} x \mathrm{p} 1,0 \mathrm{p} t \mathrm{p} 20$ and $t=0.2$ for the $2 \mathrm{D}$ graphics.

Figure 2. the plot 3D and 2D surfaces of Eq. (3.11) by considering the values:

$a_{1}=0.1, a_{2}=0.5, \mu=1, \lambda=1, \beta=1, w=2, B=0.3, c_{1}=0.8, c_{2}=1, A_{0}=0.9, A_{1}=3$,

$-1 \mathrm{p} x \mathrm{p} 1,0 \mathrm{p} t \mathrm{p} 20$ and $t=0.2$ for the $2 \mathrm{D}$ graphics.

Figure 3. the plot 3D and 2D surfaces of Eq. (5.14) by considering the values:

$a_{1}=0.1, a_{2}=0.5, \mu=1, \lambda=1, \beta=1, w=1, B=0.3,-10 \mathrm{p} x \mathrm{p} 10,0 \mathrm{p} t \mathrm{p} 20$ and $t=0.2$

for the $2 \mathrm{D}$ graphics. 
Figure 4. the plot 3D and 2D surfaces of Eq. (5.14) by considering the values: $a_{1}=0.1, a_{2}=0.5, \mu=1, \lambda=1, \beta=1, w=1, B=0.3,-10 \mathrm{p} \times \mathrm{p} 10,0 \mathrm{p} t \mathrm{p} 20$ and $t=0.2$ for the $2 \mathrm{D}$ graphics.

Figure 5. the plot 3D and 2D surfaces of Eq. (5.15) by considering the values: $a_{1}=0.1, a_{2}=0.5, \mu=1, \lambda=1, \beta=1, w=1, B=-0.3,-10 \mathrm{p} \times \mathrm{p} 10,0 \mathrm{p} t \mathrm{p} 20$ and $t=0.2$ for the $2 \mathrm{D}$ graphics.

Figure 6. the plot $3 \mathrm{D}$ and $2 \mathrm{D}$ surfaces of Eq. (5.15) by considering the values: $a_{1}=0.1, a_{2}=0.5, \mu=1, \lambda=1, \beta=1, w=1, B=-0.3,-10 \mathrm{p} x \mathrm{p} 10,0 \mathrm{p} t \mathrm{p} 20$ and $t=0.2$ for the $2 \mathrm{D}$ graphics.

Figure 7. the plot 3D and 2D surfaces of Eq. (7.4) by considering the values: $a_{1}=0.1, a_{2}=0.5, \mu=1, \lambda=1, \beta=1, w=2, B=0.3, c_{1}=0.8, c_{2}=1, A_{0}=0.9, A_{1}=3$, $-1 \mathrm{p} x \mathrm{p} 1,0 \mathrm{p} t \mathrm{p} 20$ and $t=0.2$ for the $2 \mathrm{D}$ graphics.

Figure 8. the plot 3D and 2D surfaces of Eq. (7.5) by considering the values: $a_{1}=0.1, a_{2}=0.5, \mu=1, \lambda=1, \beta=1, w=2, B=0.3, c_{1}=0.8, c_{2}=1, A_{0}=0.9, A_{1}=3$, $-1 \mathrm{p} x \mathrm{p} 1,0 \mathrm{p} t \mathrm{p} 20$ and $t=0.2$ for the $2 \mathrm{D}$ graphics.

Figure 9. the plot 3D and 2D surfaces of Eq. (7.7) by considering the values: $a_{1}=0.1, a_{2}=0.5, \mu=1, \lambda=1, \beta=1, w=2, B=0.3, c_{1}=0.8, c_{2}=1, A_{0}=0.9, A_{1}=3$, $-1 \mathrm{p} x \mathrm{p} 1,0 \mathrm{p} t \mathrm{p} 20$ and $t=0.2$ for the 2D graphics.

Figure 10. the plot 3D and 2D surfaces of Eq. (7.8) by considering the values: $a_{1}=0.1, a_{2}=0.5, \mu=1, \lambda=1, \beta=1, w=2, B=0.3, c_{1}=0.8, c_{2}=1, A_{0}=0.9, A_{1}=3$, $-1 \mathrm{p} x \mathrm{p} 1,0 \mathrm{p} t \mathrm{p} 20$ and $t=0.2$ for the $2 \mathrm{D}$ graphics. 
Figures
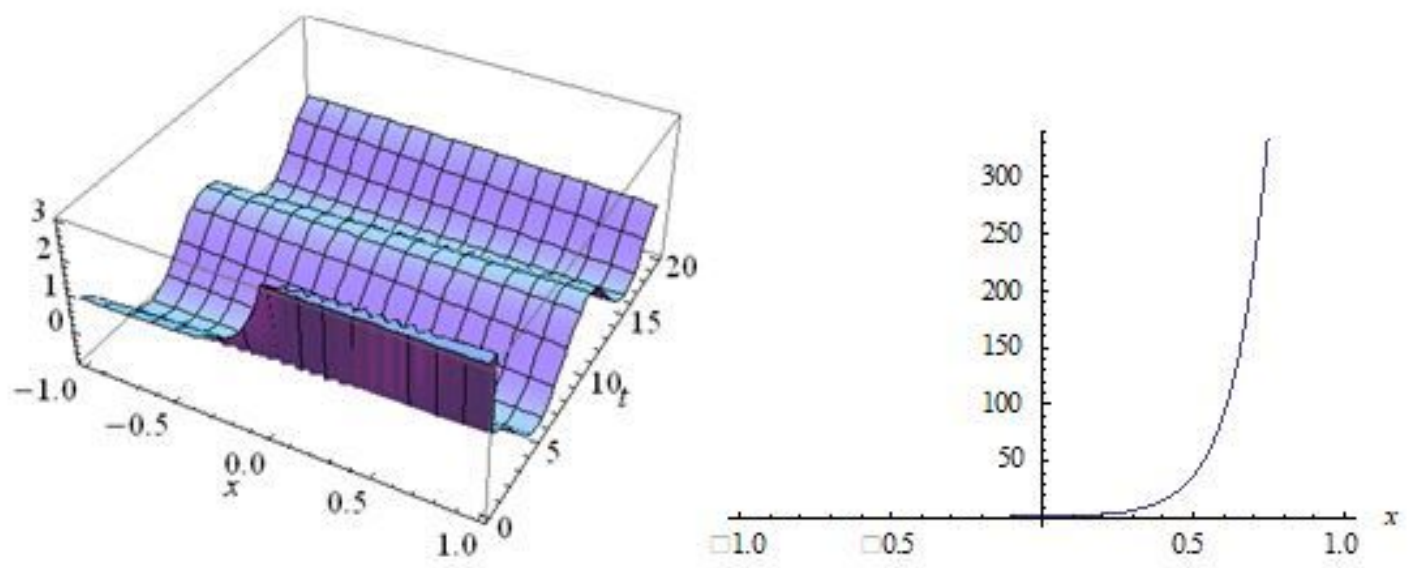

Figure 1

Figure 1


Figure 2

Figure 2 

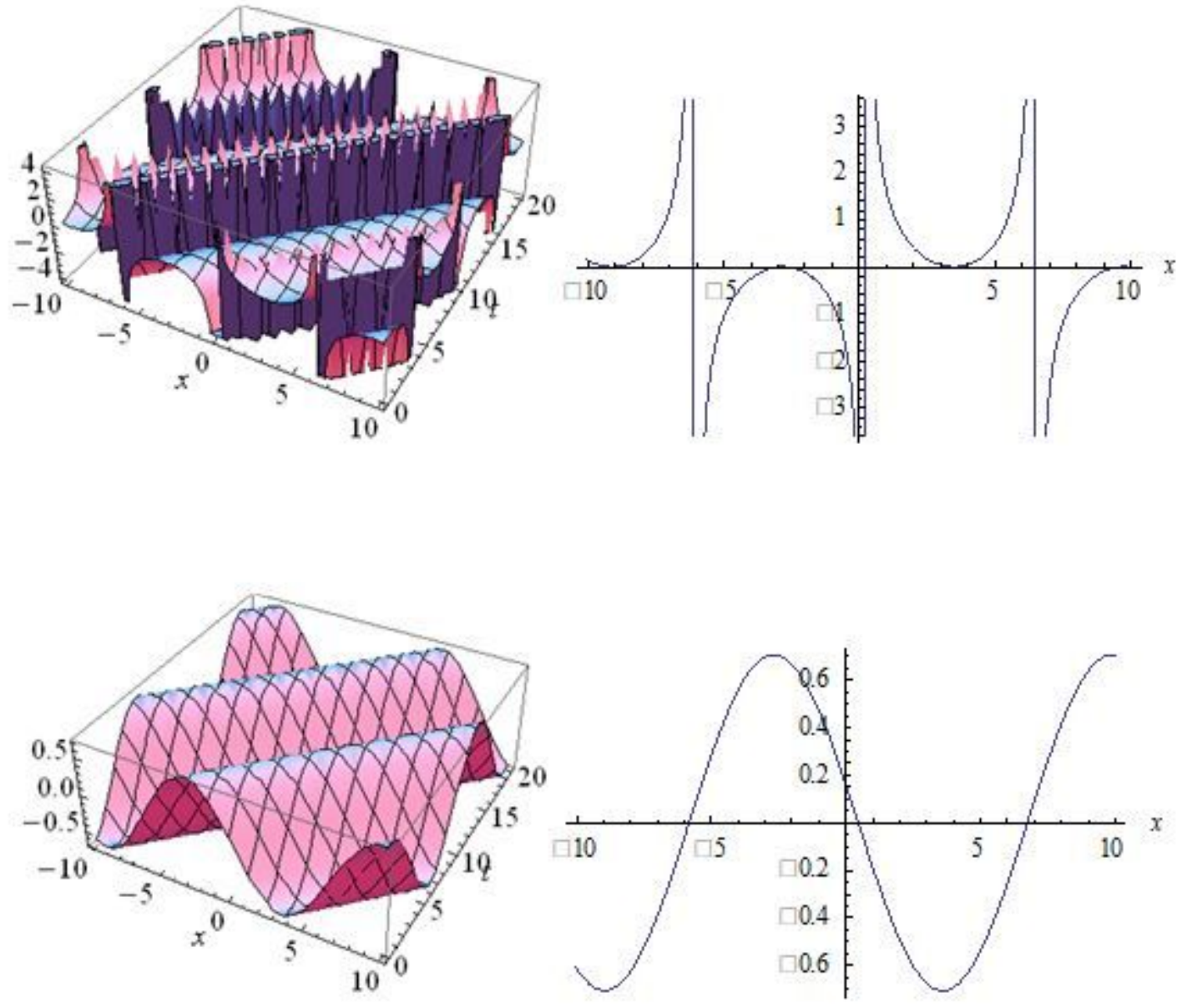

Figure 3

Figure 3 

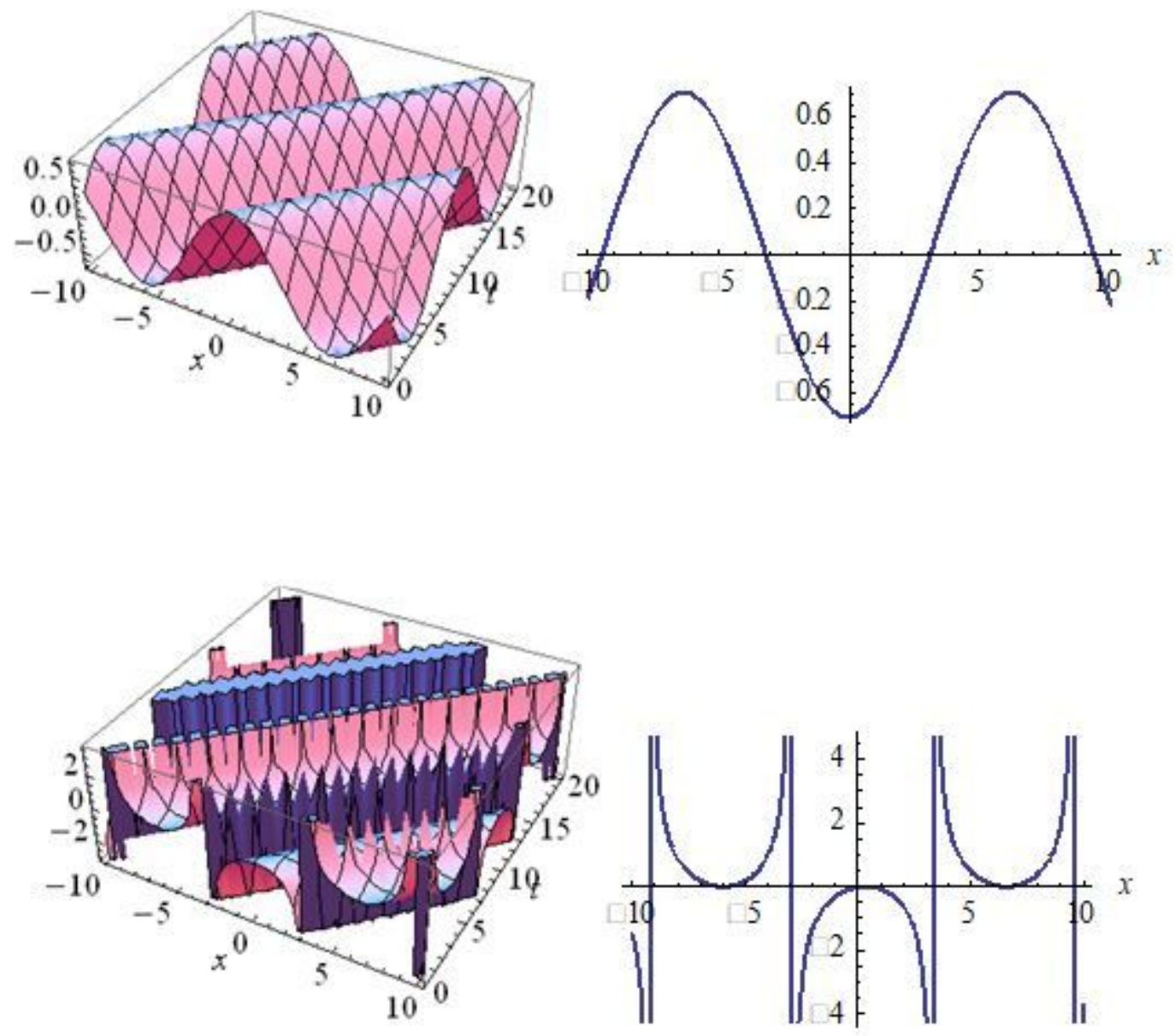

Figure 4

Figure 4 

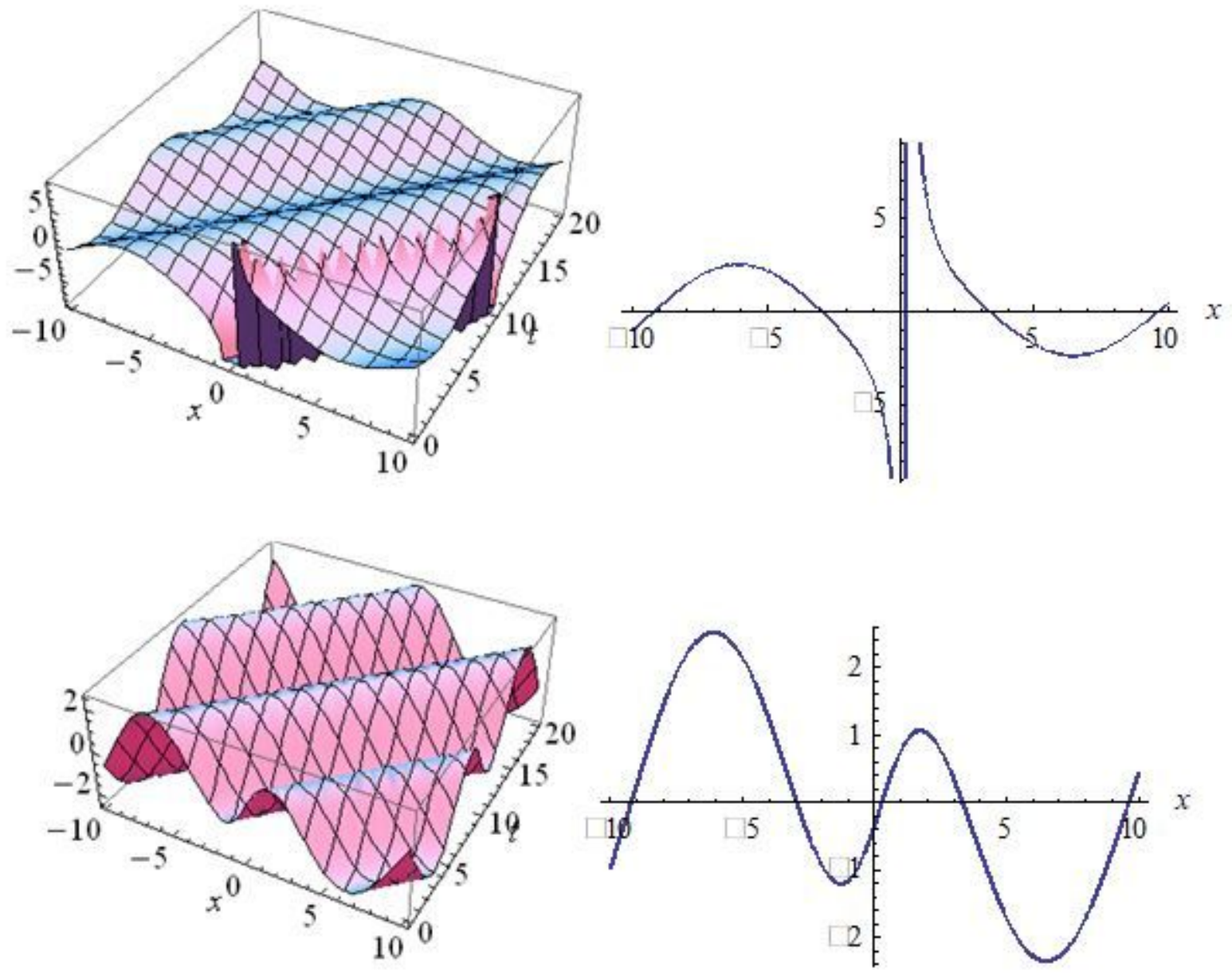

Figure 5

Figure 5 

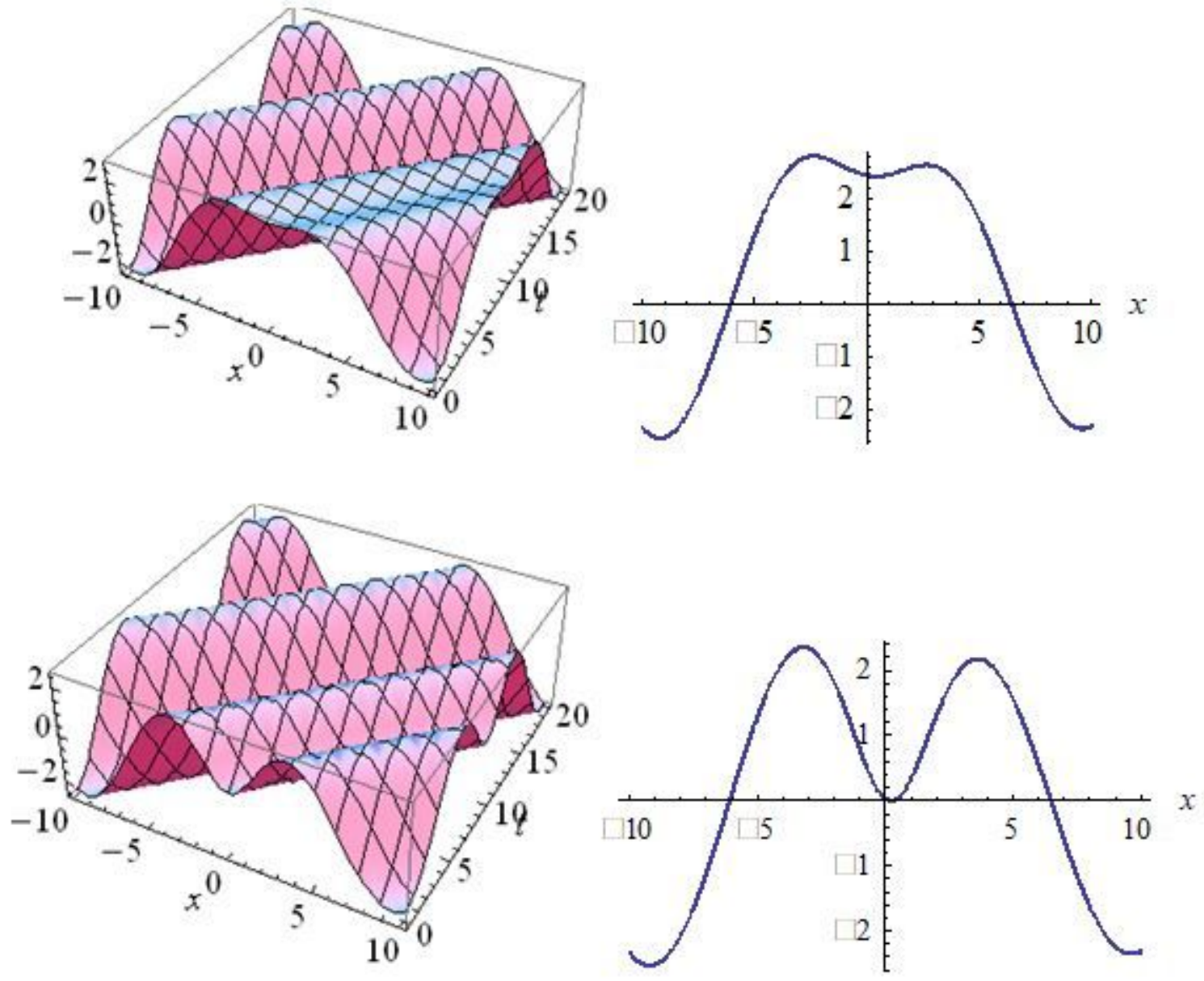

Figure 6

Figure 6
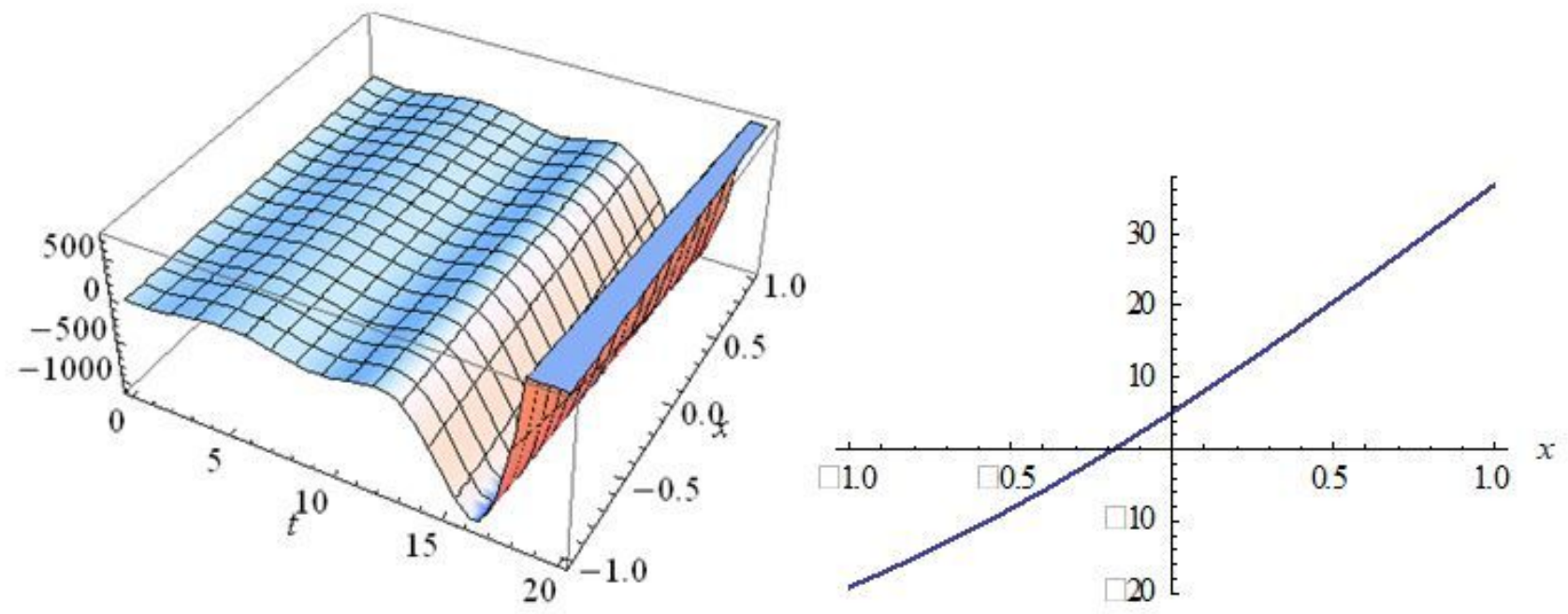

Figure 7 
Figure 7
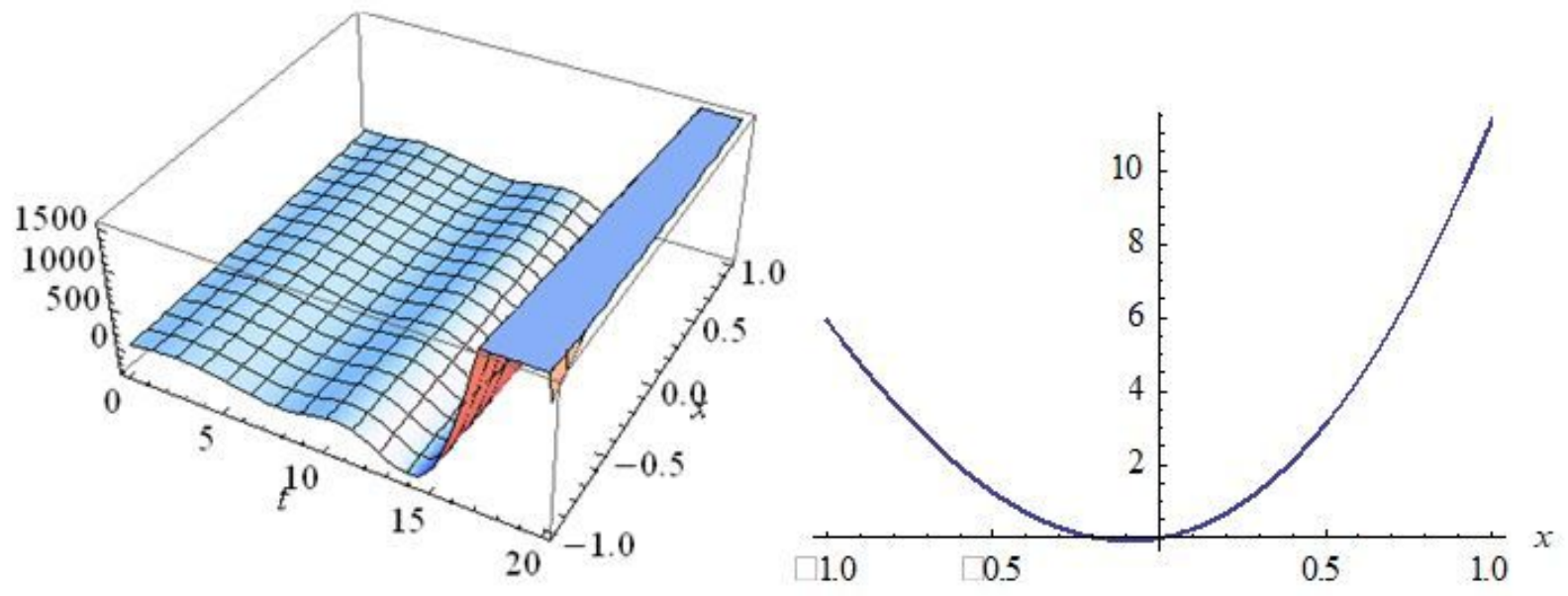

Figure 8

Figure 8
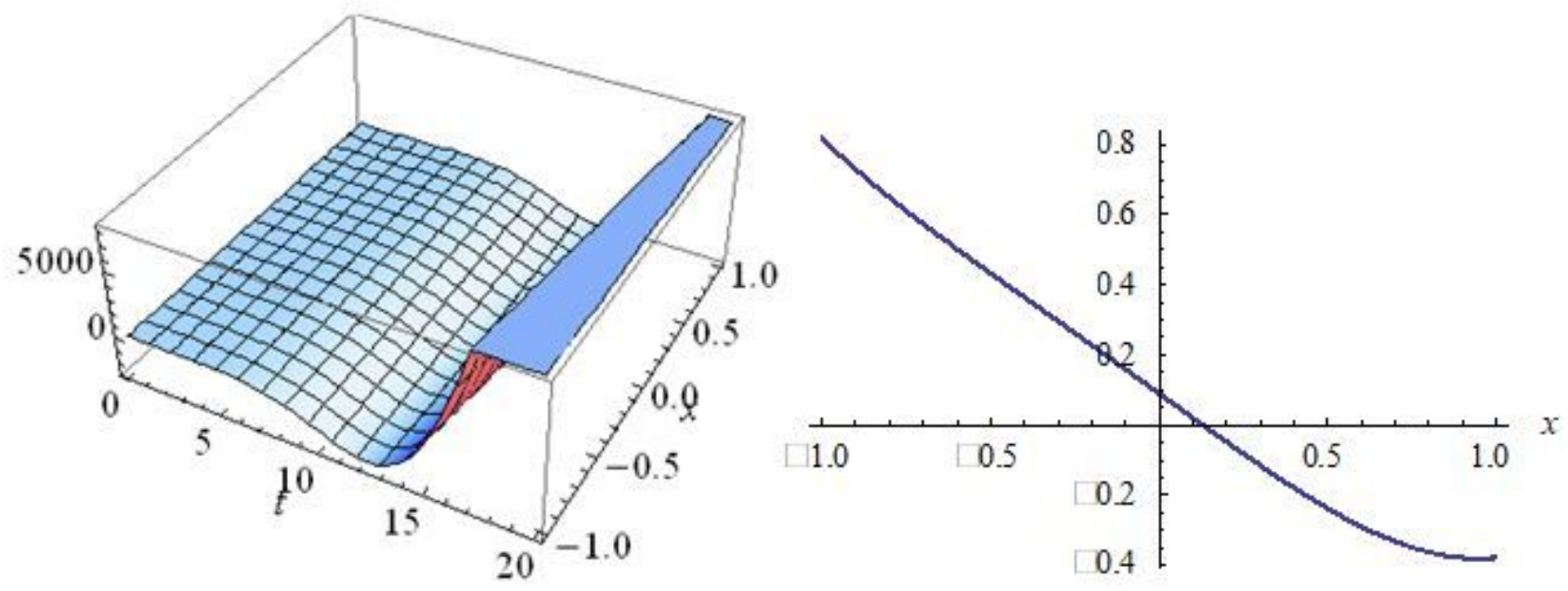

Figure 9

Figure 9 

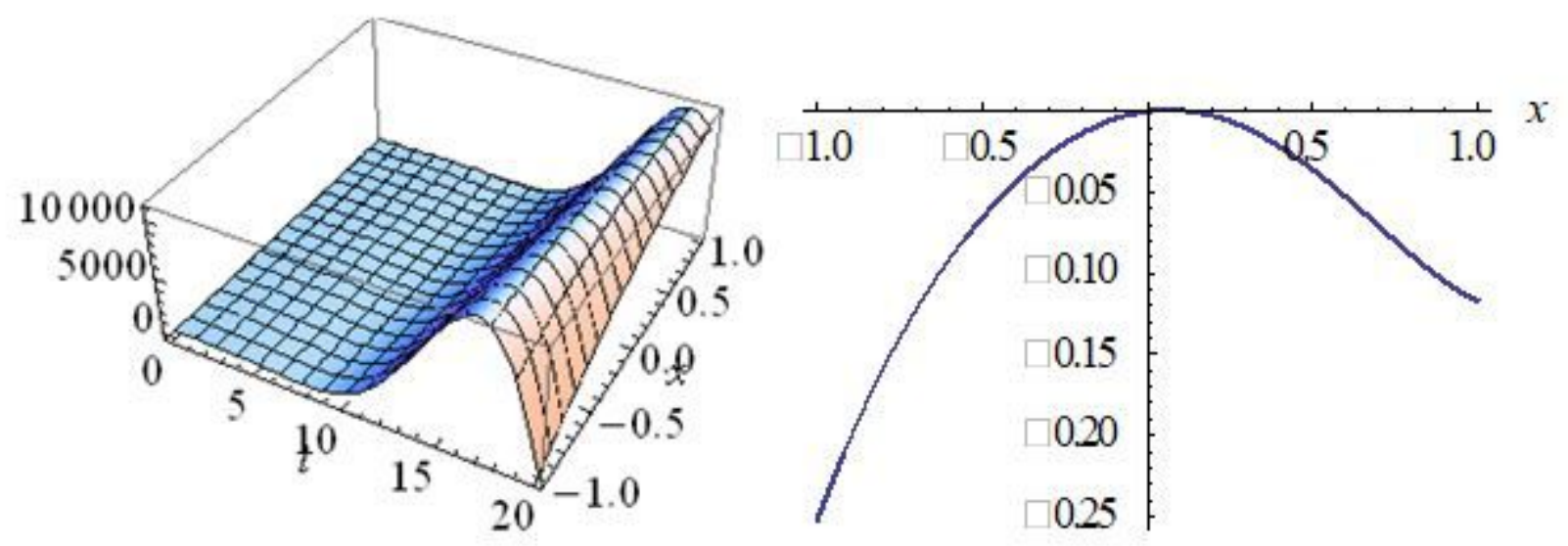

Figure 10

Figure 10 